\title{
Three restricted forms of Epstein-Barr virus latency counteracting apoptosis in c-myc-expressing Burkitt lymphoma cells
}

\author{
Gemma L. Kelly, Anne E. Milner, Gouri S. Baldwin, Andrew I. Bell, and Alan B. Rickinson* \\ Cancer Research UK Institute for Cancer Studies, University of Birmingham, Edgbaston, Birmingham B15 2TT, United Kingdom
}

Edited by Elliott D. Kieff, Harvard University, Boston, MA, and approved August 10, 2006 (received for review November 17, 2005)

Epstein-Barr virus (EBV), a human herpesvirus, transforms B cell growth in vitro through expressing six virus-coded Epstein-Barr nuclear antigens (EBNAs) and two latent membrane proteins (LMPs). In many EBV-associated tumors, however, viral antigen expression is more restricted, and the aetiological role of the virus is unclear. For example, endemic Burkitt lymphoma (BL) classically presents as a monoclonal, c-myc-translocation-positive tumor in which every cell carries EBV as an EBNA1-only (Latency I) infection; such homogeneity among EBV-positive cells, and the lack of EBV-negative comparators, hampers attempts to understand EBV's role in BL pathogenesis. Here, we describe an endemic BL that was unusually heterogeneous at the single-cell level and, in early passage culture, yielded a range of cellular clones, all with the same c-myc translocation but differing in EBV status. Rare EBV-negative cells were isolated alongside EBV-positive cells displaying one of three forms of restricted latency: (i) conventional Latency I expressing EBNA1 only from a WT virus genome, (ii) Wp-restricted latency expressing EBNAs 1, 3A, 3B, 3C, and -LP only from an EBNA2-deleted genome, and (iii) a previously undescribed EBNA2 ${ }^{+}$/LMP1 - latency in which all six EBNAs are expressed again in the absence of the LMPs. Interclonal comparisons showed that each form of EBV infection was associated with a specific degree of protection from apoptosis. Our work suggests that EBV acts as an antiapoptotic rather than a growth-promoting agent in BL by selecting among three transcriptional programs, all of which, unlike the full virus growth-transforming program, remain compatible with high c-myc expression.

viral lymphomagenesis | cell survival | viral genome integration | viral transformation

둘 pstein-Barr virus (EBV) is a B lymphotropic herpesvirus linked to several B cell malignancies $(1,2)$. In vitro the virus transforms human B cells into permanent lymphoblastoid cell lines (LCLs) expressing the full range of virus-coded latent proteins; the nuclear antigens EBNAs 1, 2, 3A, 3B, 3C, and -LP are expressed from adjacent promoters $\mathrm{Cp}$ and $\mathrm{Wp}$ in the BamHI $\mathrm{C}$ and $\mathrm{W}$ regions of the genome, and the latent membrane proteins LMPs 1 and 2 from independent EBNA2activated promoters in the BamHI $\mathrm{N}$ region (3). This same growth transforming function also is apparent in vivo and can lead to uncontrolled cell growth. Thus, most cases of EBVassociated posttransplant lymphoproliferative disease show the same latent gene expression profile as LCLs (Latency III) and appear to represent directly virus-transformed lesions growing out in the absence of host $\mathrm{T}$ cell surveillance. By contrast, other EBV-positive B cell malignancies such as Burkitt lymphoma (BL) show more restricted forms of latent gene expression, and the virus' contribution to lymphomagenesis is less well understood $(1,2)$.

BL occurs as a high incidence "endemic" tumor in Africa and New Guinea, where it is consistently EBV-positive, and elsewhere as a lower incidence "sporadic" disease that is less frequently linked to EBV (2). In all BLs, irrespective of EBV status, the key growth-transforming event appears to be consti- tutive activation of the c-myc oncogene through its translocation into an Ig gene locus (4). Most EBV-positive BLs express just one viral protein, EBNA1, from an alternative promoter Qp in the BamHI Q region of the genome (Latency I) $(3,5)$, prompting debate as to how EBNA1 or, indeed, the noncoding EBER RNAs (present in all forms of latency), might contribute to BL pathogenesis $(6-10)$. Although such restricted viral antigen expression potentially reduces tumor immunogenicity, this form of latency may have arisen during tumor evolution for reasons other than immune evasion. Thus, in in vitro systems, enforcing the full Latency III program in a BL cell background proved to be incompatible with high c-myc expression and with maintenance of the malignant BL cell phenotype $(11,12)$.

Recently, we identified BLs with a different form of restricted virus latency, again compatible with high c-myc expression but involving $\mathrm{Wp}$ rather than $\mathrm{Qp}$ usage and characterized by expression of EBNAs 1, 3A, 3B, 3C, and -LP in the absence of EBNA2 and the LMPs (13). These "Wp-restricted" tumors had arisen through infection of the tumor progenitor cell with an EBNA2 gene-deleted virus, leading to $\mathrm{Wp}$-initiated rather than Qp-initiated transcription. Such selection for a rare EBNA2 gene deletion suggested that expression of EBNA2, or the EBNA2-induced LMP genes, was the source of incompatibility between the virus-driven and c-myc-driven growth programs.

In contrast to Latency I and Wp-restricted tumors, all of which are homogeneous at the single-cell level $(5,14)$, here we describe an EBV-positive endemic BL in which the tumor clone contains some cells displaying Latency I, others Wp-restricted latency, and others a unique EBNA2-positive, LMP-negative form of infection. Comparing these cells with rare EBV genome-loss clones derived from the same tumor shows how each form of latency is associated with a different degree of protection from apoptosis.

\section{Results}

EBV Genome Content of Awia-BL. Screening endemic BL biopsies by DNA PCR with different EBV primer/probe combinations identified one tumor, Awia-BL, as carrying both an amplifiable WT EBNA2 gene sequence and a BamHI W/H fusion fragment diagnostic of EBNA2 gene deletion (Fig. 1A). Sequencing revealed a deletion whose boundaries mapped close to those already seen in the Wp-restricted tumors Sal-BL, Oku-BL, and Ava-BL (Fig. 1B). The Awia-BL biopsy cells grew immediately in vitro, with first passage cells cryopreserved within 7 days, and

Author contributions: G.L.K. and A.B.R. designed research; G.L.K. and G.S.B. performed research; A.E.M. and A.I.B. contributed new reagents/analytic tools; G.L.K. analyzed data; and G.L.K. and A.B.R. wrote the paper.

The authors declare no conflict of interest.

This paper was submitted directly (Track II) to the PNAS office.

Abbreviations: BL, Burkitt lymphoma; EBNA, Epstein-Barr nuclear antigen; LCL, lymphoblastoid cell line; LMP, latent membrane protein.

*To whom correspondence should be addressed. E-mail: a.b.rickinson@bham.ac.uk.

(c) 2006 by The National Academy of Sciences of the USA 
A

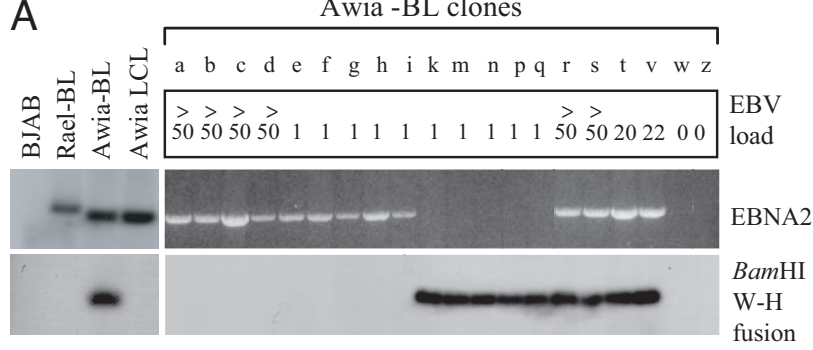

B

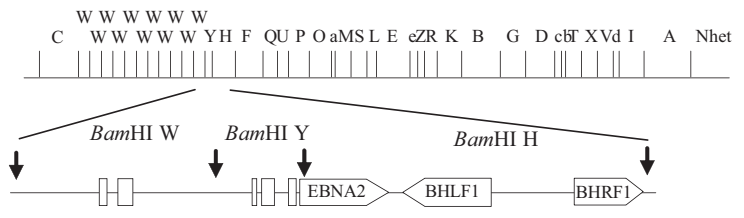

Awia-BL

Sal-BL

Oku-BL

Ava-BL

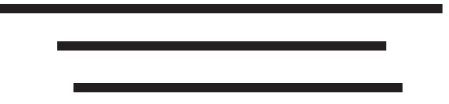

45426-52816

$45297-53836$

45793-52546

C

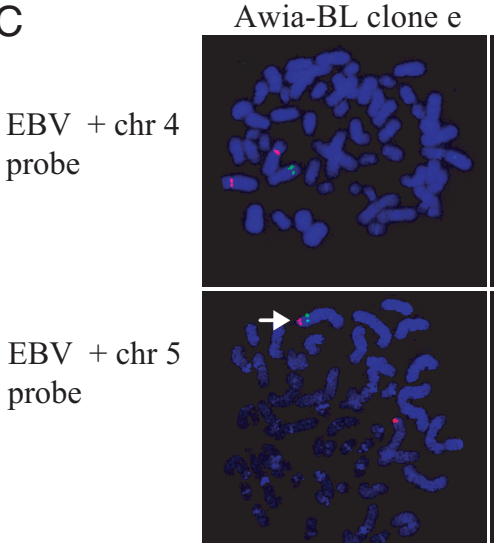

Awia-BL clone m

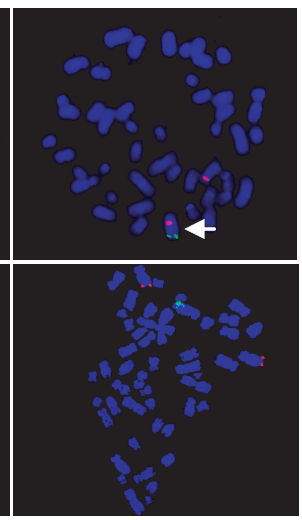

Fig. 1. EBV genome content of Awia-BL. (A Left) $P C R$ analysis of Awia-BL and the Awia-tr-LCL by using primer/probe combinations specific for the EBNA2 sequence or for a BamHI W-H fusion fragment (EBNA2 gene deletion). BJAB is an EBV-negative control line. Rael-BL is a standard Latency I BL line. (A Right) Parallel data from a representative range of Awia-BL clones. The boxed numbers refer to the total EBV genome load for each clone as determined by quantitative PCR assay for the EBV pol gene. (B) Location of the EBNA2 gene deletion in Awia-BL shown below a linearized map of the EBV genome with BamHI restriction fragments. Deletion coordinates (numbered according to the $B 95.8$ prototype strain sequence) are shown relative to those found in the Wp-restricted Sal-BL, Oku-BL, and Ava-BL lines (13). (C) Metaphase spreads of Awia-BL clone e (carrying a single EBNA2-positive genome only) and clone $\mathrm{m}$ (carrying a single EBNA2-deleted genome only) after FISH staining with a fluorescein-labeled EBV probe (green) and a spectrum-orange-labeled probe specific either for a chromosome 4 centromeric region or for a chromosome 5 telomeric region (red). Clone e shows integration on chromosome 5; clone $\mathrm{m}$ shows integration on chromosome 4 (see white arrows).

gave a cell line with a single-cell growth pattern, germinal center-like surface phenotype, and $t(8: 14)$ chromosomal translocation, all typical of BL. This Awia-BL line retained the same amplifiable EBNA2 and BamHI $\mathrm{W} / \mathrm{H}$ fusion sequences as the original tumor, the presence of a WT genome being confirmed by inducing virus replication and selectively rescuing that genome into normal B cells to produce the Awia tr-LCL (Fig. 1A).

We then established 200 Awia-BL clones by limiting dilution of early passage cultures in acyclovir-containing medium, screened all clones for total EBV genome load by using a quantitative PCR assay amplifying EBV pol gene sequences, and then screened a selection of clones for EBNA2-positive (WT) and EBNA2-deleted status as above. This identified 2 from 200 clones that were consistently EBV genome-negative and (from 70 EBV-positive clones analyzed in full) 50 clones carrying multiple copies of the WT genome only, 6 clones carrying a single WT genome only, 6 clones carrying a single EBNA2deleted genome only, and 8 clones carrying both types of genome; representative results are shown in Fig. $1 A$. All clones were derived clearly from the same malignant population in vivo because, where analyzed, all carried the same productive $\mathrm{IgH}$ gene rearrangement and, in addition to the $\mathrm{t}(8: 14)$ translocation, a trisomy chromosome 20 marker that was distinctive of this particular tumor.

The above interclonal differences in EBV genome load were confirmed (data not shown) by using FISH with an EBV probe capable of detecting individual virus genomes (15). We then focused on those clones with single EBV genomes and found that they fell into two groups, each with a different integration event. Fig. $1 C$ shows representative chromosome spreads dually hybridized with a fluorescein-labeled EBV probe and with a spectrum-orange-labeled chromosome-specific probe; consistently, clones with a single WT virus genome (e.g., clone e) showed integration of that genome on chromosome 5, and clones with a single EBNA2-deleted genome (e.g., clone m) showed integration on chromosome 4.

EBV Latency in the Awia-BL Cell Line and Derived Clones. The Awia-BL line and derived clones then were assayed by quantitative RT-PCR for Wp-initiated transcripts (typically seen at high levels in Wp-restricted latency and at low levels in Latency III), Cp-initiated transcripts (diagnostic of Latency III), Qpinitiated EBNA1 transcripts (diagnostic of Latency I), and EBNA2, LMP1, and LMP2 gene-specific mRNAs. Transcription data from the parental Awia-BL line and representative clones are presented alongside the corresponding Awia tr-LCL values in Fig. 2; LMP2 mRNA levels are not shown because they are generally very low. The same cells also were analyzed by immunoblotting for expression of the EBNA1, EBNA2, EBNA3A, LMP1, and c-myc proteins. Representative results are presented in Fig. 3, with $\beta$-actin immunoblots as loading controls. The patterns observed were the following:

Awia-BL vs. Awia tr-LCL. The parental Awia-BL line was unlike any previously studied Burkitt line. It was strongly positive for Qp-initiated EBNA1 transcripts (typical of Latency I) but also showed some Wp activity in the absence of Cp (suggestive of Wp-restricted latency) plus the unexpected combination of EBNA2 transcription in the absence of LMP1 mRNA. By contrast, the Awia tr-LCL showed a characteristic Latency III pattern with $\mathrm{Cp}$ dominant over $\mathrm{Wp}$, with both EBNA2 and LMP1 transcripts strongly expressed and with no detectable Qp activity (Fig. 2). At the level of antigen expression, as expected, the Awia tr-LCL was positive for EBNAs 1,2,3A, and LMP1 and showed the same low level of c-myc protein as the X50-7 and $\mathrm{C} 2+\mathrm{BL} 16$ reference LCLs. Note that the Awia-virus isolate is a type 2 virus strain and, therefore, encodes an EBNA2 protein of similar size to that seen in the reference type 2 LCL, C2+BL16 (5). Awia-BL gave a unique protein profile. It was EBNA1positive but also contained low levels of EBNA2 and EBNA3A in the absence of any detectable LMP1 and expressed c-myc at the high levels typical of classical BL (Fig. 3).

EBV-negative BL clones. The two rare clones (w and $\mathrm{z}$ ) originally identified as EBV genome-negative indeed were completely lacking in detectable viral transcripts or viral proteins (Figs. 2 and 3 ).

Latency I BL clones. All clones carrying multiple copies of only the WT genome displayed Latency I infection. Such clones (represented by a-d) showed high-level Qp activity with barely de- 


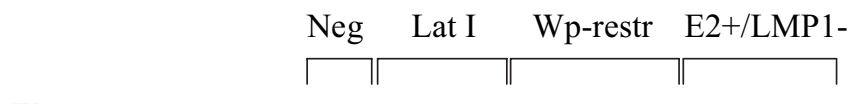

$\mathrm{Wp}$

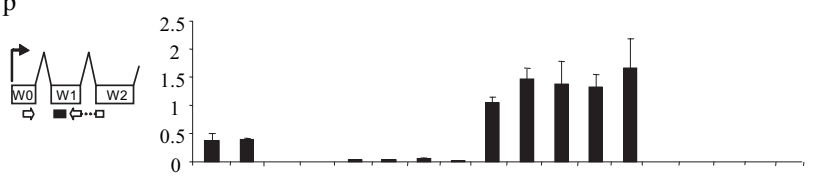

$\mathrm{Cp}$

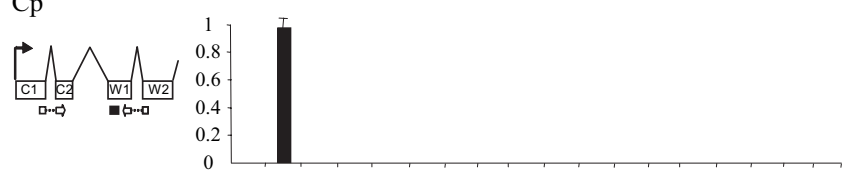

Qp EBNA1
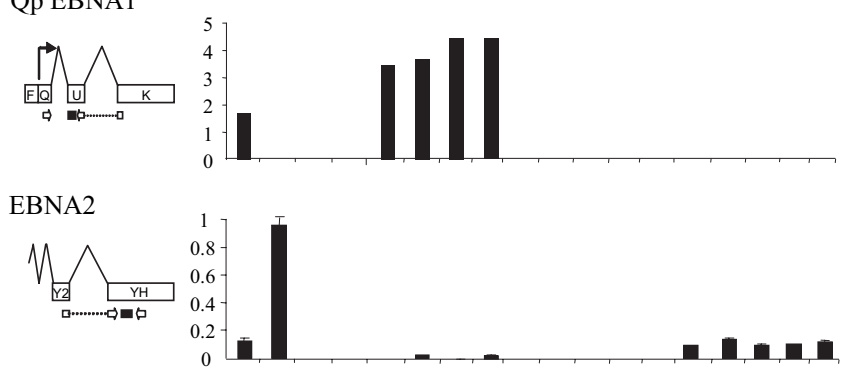

LMP1

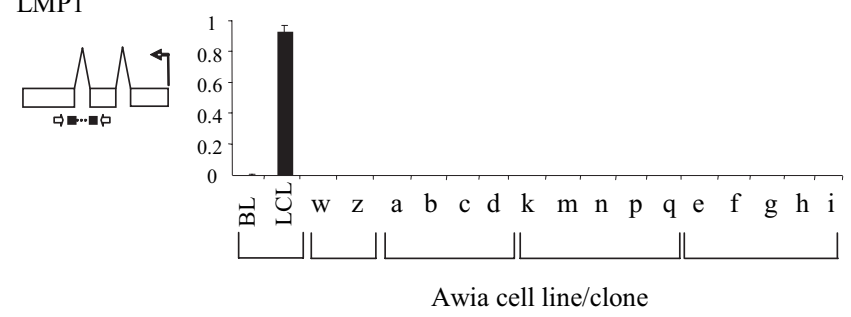

Fig. 2. Analysis of EBV latent transcription in the parental Awia-BL line, the Awia tr-LCL, and representative EBV genome-negative, Latency I, Wp-restricted, and EBNA2 ${ }^{+} / \mathrm{LMP1}^{-}$Awia-BL clones. Transcript levels (with transcript-specific primer-probe combinations as shown) relative to an appropriate positive control line (assigned a value of 1). Note that the BamHI Q-U-K-spliced transcript levels indeed reflected Qp usage because there was no detectable F-Q-U-K-spliced transcripts from the lytic Fp promoter (data not shown).

tectable expression of any other latent cycle transcripts (Fig. 2). Accordingly, the cells expressed EBNA1 in the absence of EBNA2, EBNA3A, and LMP1 and displayed typically high levels of c-myc (Fig. 3).

Wp-restricted BL clones. All clones containing only an EBNA2deleted genome (clones $\mathrm{k}-\mathrm{q}$ ) displayed classical features of $\mathrm{Wp}$-restricted latency. All had high levels of Wp-initiated transcripts in the complete absence of Cp-, Qp-, EBNA2, and LMP1 transcription (Fig. 2), expressed EBNA1 and EBNA3A in the absence of detectable EBNA2 and LMP1, and retained high c-myc levels (Fig. 3). Clones containing both EBNA2-deleted and WT genomes showed an essentially similar pattern of results (data not shown), supporting the view that in such cells, Wprestricted transcripts derive exclusively from the EBNA2deleted genome (13).

EBNA2-positive, LMP1-negative BL clones. All clones carrying only a single copy of the WT genome displayed a previously undescribed EBNA2 ${ }^{+} / \mathrm{LMP}^{-}$form of latency. Such clones (represented by e-i) were consistently negative for $\mathrm{Wp}-, \mathrm{Cp}-$, and Qp activity and were negative for LMP1 transcripts, yet did contain significant levels of EBNA2 mRNA (Fig. 2). At the protein level, these clones were positive for EBNA1, EBNA2, and EBNA3A

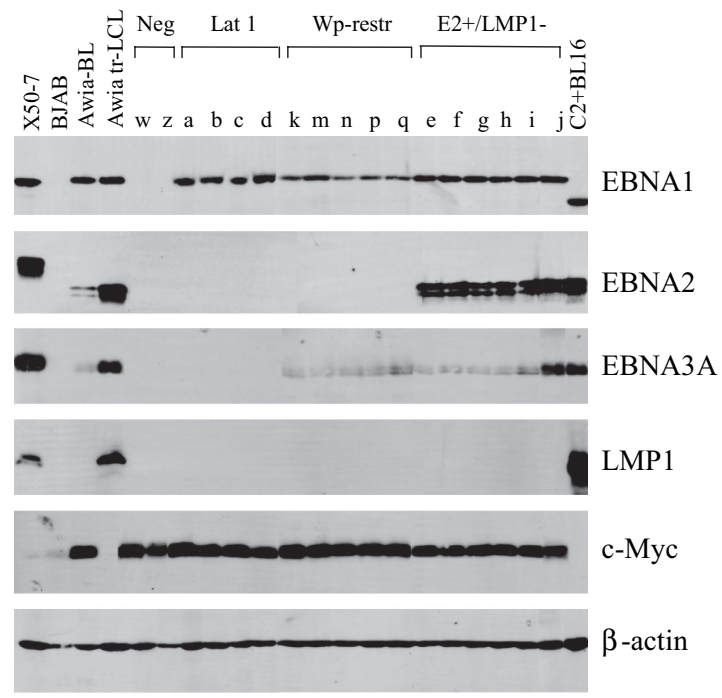

Fig. 3. Analysis of EBV latent protein expression in the parental Awia-BL line, the Awia tr-LCL, and representative Awia-BL clones as in Fig. 2. Results are presented as immunoblots probed with antibodies specific for EBNA1, EBNA2, EBNA3A, and LMP1. Positive control lines were the type 1 virus-transformed X50-7 LCL and the type 2 virus-transformed $\mathrm{C} 2+\mathrm{BL} 16 \mathrm{LCL} ; \mathrm{BJAB}$ is the EBVnegative control line. Immunoblots of the same samples were probed with antibodies against c-myc and, as a loading control, against $\beta$-actin. Note that by immunoblotting levels of EBNA2 in the EBNA2 ${ }^{+} / \mathrm{LMP}^{-}$- clones was estimated to be $20-60 \%$ of that seen in the C2+BL16 LCL.

but negative for LMP1. Importantly, they expressed the same high levels of c-myc as did the Latency I and Wp-restricted clones (Fig. 3). Moreover, cells with this unique form of infection indeed were present in the original tumor because monoclonal antibody staining of the Awia-BL biopsy (data not shown) and the Awia-BL line at its first in vitro passage (Fig. 4) identified $5-10 \%$ cells, giving strong EBNA2 staining in the absence of LMP1. Staining of Awia-BL clones identified some clones (e.g., clone e) with homogeneous low-level staining and other clones (e.g., clone j) with a greater range of intensities, including some cells with almost LCL-like levels (compare Awia tr-LCL). Importantly, all cells within these EBNA2-positive clones remained negative for LMP1 staining.

EBV Latency and BL Cell Susceptibility to Apoptosis. One of the cardinal features of the Burkitt tumor is a marked sensitivity to apoptosis (16), thought to be a consequence of deregulated

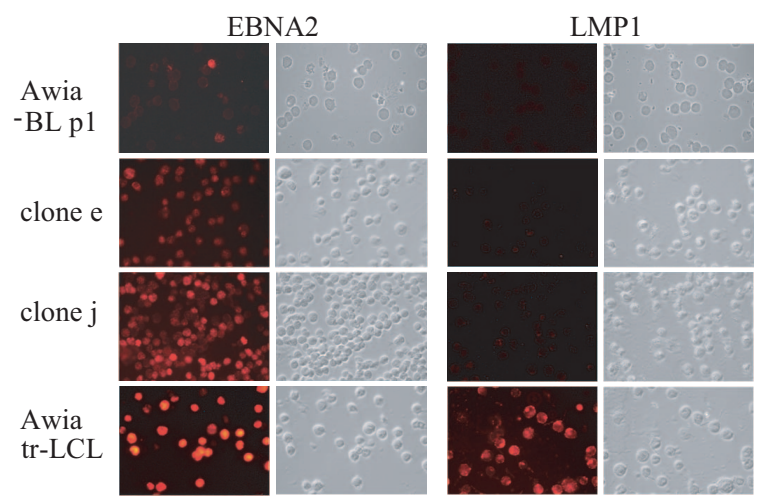

Fig. 4. EBNA2 and $L M P 1$ staining of Awia-BL (at the first in vitro passage within 7 days of tumor cell culture), two EBNA2 ${ }^{+} /$LMP1 $^{-}$Awia-BL clones (e and j), and the Awia tr-LCL. Immunofluorescence results are shown alongside bright field photomicrographs of the same area. 
A
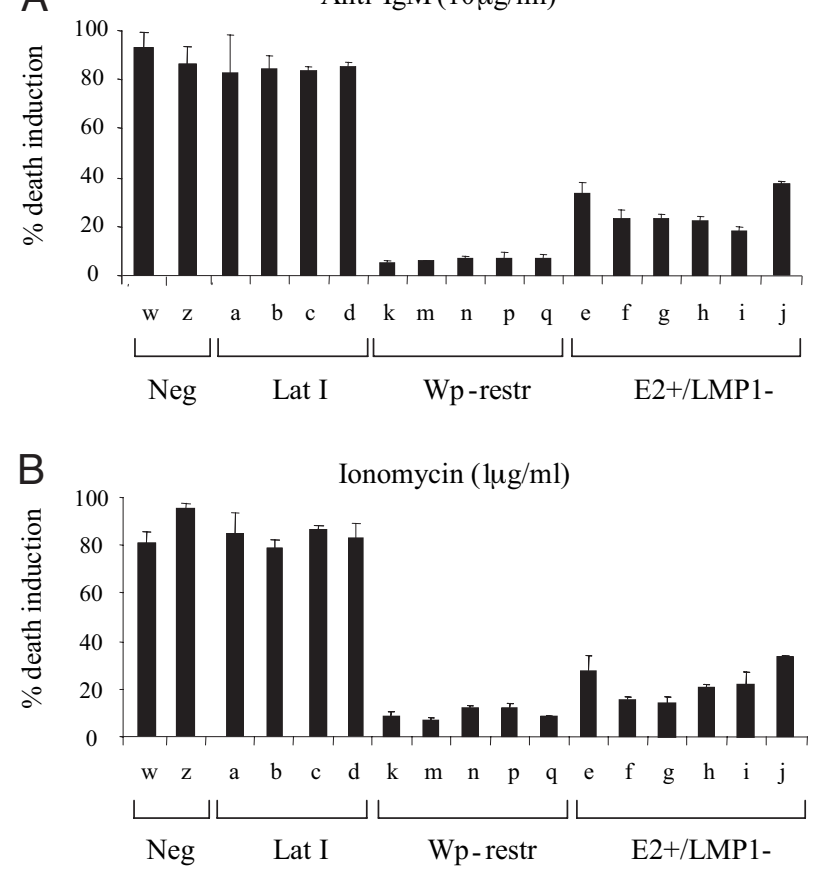

C $\quad \operatorname{Anti}-\operatorname{IgM}(2.5 \mu \mathrm{g} / \mathrm{ml})$
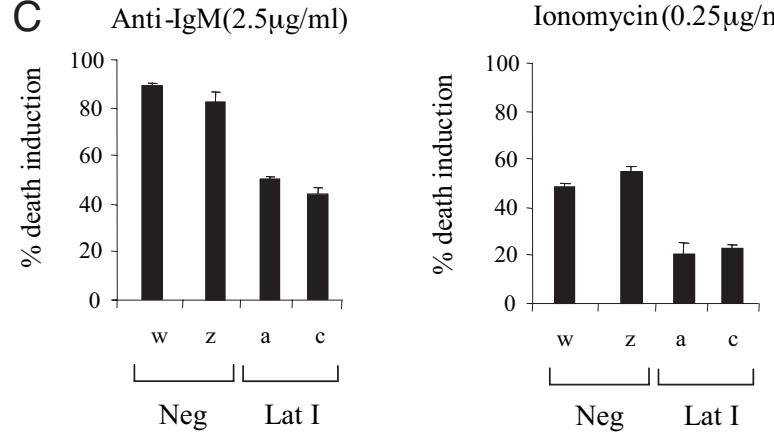

Fig. 5. Relationship among EBV status, form of EBV latency, and susceptibility to apoptosis in Awia-BL cells. $(A$ and $B$ ) Mean results from multiple apoptosis assays in which Awia-BL clones were exposed to anti-IgM at 10 $\mu \mathrm{g} / \mathrm{ml}$ for $72 \mathrm{~h}(A)$ or ionomycin at $1 \mu \mathrm{g} / \mathrm{ml}$ for $48 \mathrm{~h}(B)$ and then stained with propidium iodide and Syto 16 . Results are expressed as the percentage of cells induced to die. Extending the assay period up to $96 \mathrm{~h}$ in each case did not affect the pattern of results (data not shown). (C) Mean results from additional assays in which EBV-negative and Latency I clones were tested at lower doses of 2.5 $\mu \mathrm{g} / \mathrm{ml}$ anti-lgM and $0.25 \mu \mathrm{g} / \mathrm{ml}$ ionomycin. Note that in all experiments, essentially similar results were obtained by using caspase cleavage as a marker of apoptosis in single cells.

c-myc expression (17). A role for EBV as an antiapoptotic agent in BL pathogenesis first was implied from work with Akata-BL, an unusual sporadic BL line that is EBV-positive, but in late passage, frequently yields EBV genome-negative subclones (18); these EBV-loss subclones proved more prone to apoptosis than the parental line $(7,10)$. Here, we were able to readdress this issue with a wider variety of clones all derived from the same early passage endemic tumor. The clones were subjected to two well defined apoptotic triggers, surface IgM ligation (note that all clones showed equivalent levels of surface IgM) and ionomycin treatment (16), and responses were quantitated by Syto 16/propidium iodide dual labeling and flow cytometry (14). Fig. 5 shows composite data from repeated assays. At high doses of the inducing agents $(10 \mu \mathrm{g} / \mathrm{ml}$ anti-IgM and $1 \mu \mathrm{g} / \mathrm{ml}$ ionomycin $)$, $>80 \%$ of cells in the EBV-negative and Latency I clones died, mostly by apoptosis (Fig. $5 A$ and $B$ ). However, at lower doses,
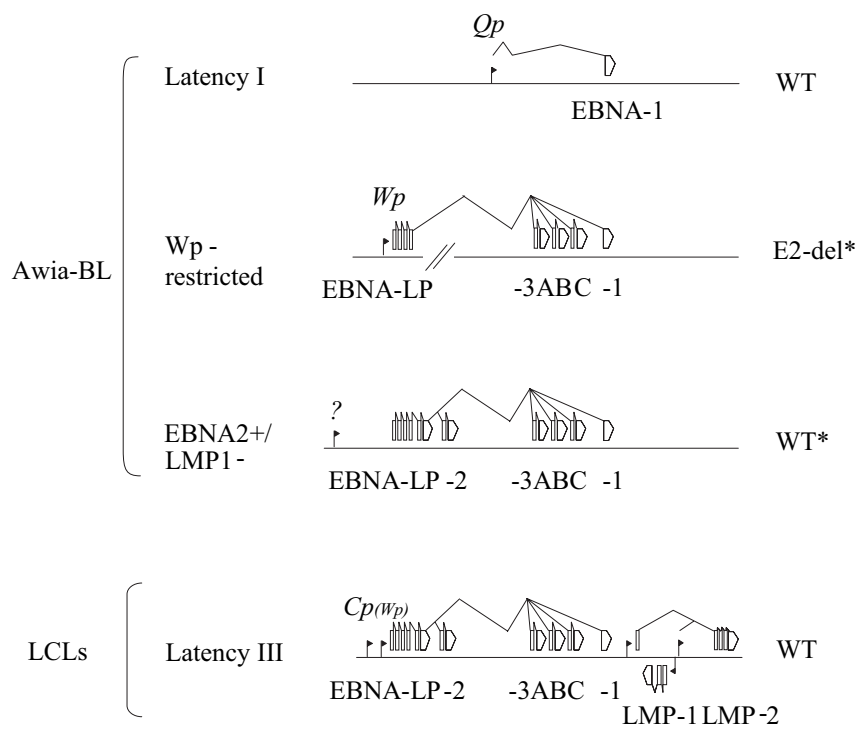

Fig. 6. Diagrammatic representation of the three different forms of restricted EBV latency found in the Awia-BL tumor compared with the standard Latency III infection typical of all LCLs. Note that the Latency I and EBNA2+/ LMP1- forms of latency involve different patterns of transcription from an EBNA2 gene-positive WT EBV genome, whereas the Wp-restricted form of latency involves transcription from an EBNA2 gene-deleted genome. In Awia$\mathrm{BL}$, the Wp-restricted and EBNA2 $2^{+} / \mathrm{LMP1} 1^{-}$latent infections have involved the integration of single EBV genomes into chromosomes 4 and 5, respectively.

we consistently observed that Latency I clones were relatively protected compared with clones lacking the EBV genome (Fig. $5 C)$. By contrast, the other forms of latency showed clear differences even in the high-dose assays. Thus, Wp-restricted clones were much more resistant to apoptosis, with levels of cell death always in the 5-15\% range, whereas the EBNA2 ${ }^{+} / \mathrm{LMP}^{-}$ clones were intermediate between the two extremes, with 15$40 \%$ cells dying. All three EBV gene programs therefore were associated with a specific survival advantage.

\section{Discussion}

Endemic BL is the prime example of a tumor whose pathogenesis involves complementation between a transforming virus and a defined cellular genetic change. Identifying what EBV contributes to BL development has been difficult because endemic tumors typically present as a homogeneous c-myc-translocationpositive population, with every cell carrying the virus and displaying the same form of virus latency $(5,14,19)$. As a result, one does not have access to EBV-negative cells from the same tumor as comparators, the only exceptions being rare cases of EBV-loss clones arising in a tumor line after many years in culture (20). The mosaic nature of the Awia-BL tumor at presentation, with evidence of rare EBV-negative cells and cells displaying different forms of EBV infection, therefore provided a unique opportunity to ask how viral gene expression influences the BL cell phenotype.

The three forms of restricted latency seen among EBVpositive Awia-BL clones are shown in Fig. 6, alongside the full Latency III pattern of gene expression seen in in vitrotransformed LCLs. The Latency I clones, like most endemic BLs, carry multiple episomal copies of a WT EBV genome and express EBNA1 selectively from Qp. The Wp-restricted clones, like other recently described tumors of this type $(13,14)$, express EBNAs 1, 3A, 3B, 3C, and -LP exclusively from $\mathrm{Wp}$; however, these clones are unique in carrying a single EBNA2-deleted genome integrated on chromosome 4 rather than multiple EBNA2-deleted episomes. The third set of clones express all six 
EBNAs in the absence of LMPs and display yet another transcription program. Thus, despite the $\mathrm{Wp}$ and $\mathrm{Cp}$ promoter sequences being intact in these cells and $5^{\prime}$ RACE identifying EBNA2 transcripts with a W2/Y1/Y2/Y3 exon structure typical of Wp or Cp usage (data not shown), these promoters are silent and EBNA transcription appears to be initiating from a different, as yet unidentified, promoter. The EBNA2 ${ }^{+} / \mathrm{LMP}^{-}$antigen profile not only is previously undescribed but also unexpected because EBNA2 (including the protein encoded by type 2 strains like Awia) normally transactivates LMP1 and LMP2 expression (21-23) and has been considered the main source of incompatibility between the c-myc-driven and Latency III-driven growth programs $(12,24)$. Remarkably, these EBNA2 ${ }^{+} / \mathrm{LMP}^{-}$ clones are also derived from a progenitor with a unique integration event, here involving a single copy of an EBNA2-positive viral genome on chromosome 5 .

Viral genome integration is rare in the context of BL. However, the presence of these particular integrations in all $\mathrm{Wp}$-restricted and all EBNA2 ${ }^{+} / \mathrm{LMP}^{-}$clones from early passage cultures of Awia-BL strongly suggests that they must have occurred during evolution of the tumor in vivo. This finding raises the question whether the altered phenotypes of the above clones might be ascribed to the effects of integration per se? For the Wp-restricted clones, there is strong evidence against this interpretation. Thus, these clones carry an integrated EBNA2-deleted genome that show a pattern of viral gene expression and an enhanced survival phenotype similar to that of other BL lines carrying multiple episomal copies of an EBNA2-deleted virus (14). Moreover, we recently have infected EBV-loss and Latency I Awia-BL cells in vitro with an EBNA2-deleted recombinant virus and have generated clones that are Wp-restricted and, again, show enhanced survival in apoptosis assays (Fig. 7, which is published as supporting information on the PNAS web site). In the case of EBNA2 $2^{+}$ LMP1 ${ }^{-}$clones, we cannot exclude the possibility that this unusual form of latency is a by-product of viral genome integration per se. In that regard, however, integration has not led to any discernable EBNA2 mutation nor to the disruption of key LMP1 sequences; the EBNA2 and LMP1 genes and the LMP1 promoter region were found to be intact in these cells and identical to those present in the Awia-tr-LCL (data not shown). We consider it likely that the enhanced survival phenotype of these cells (versus Latency I clones) indeed is a result of their broader EBNA expression rather than some other consequence of viral genome integration; however, formal proof will require the development of a recombinant virus capable of establishing $\mathrm{EBNA}^{+} / \mathrm{LMP}^{-}$latency by in vitro infection.

These issues do not detract from the wider significance of the present findings for our understanding of BL pathogenesis. Uniquely, the Awia-BL tumor is heterogeneous at the single-cell level, as if caught in the act of clonal evolution. From the analysis of biopsy material, early passage cultures, and derived clones, we estimate that at presentation, the tumor contained $\approx 70 \%$ cells showing classical Latency I, 20\% cells with Wp-restriction, and $5-10 \%$ cells with an EBNA2 ${ }^{+} / \mathrm{LMP}^{-}$form of infection. Interestingly, the proportion of $\mathrm{Wp}$-restricted and EBNA2 ${ }^{+} / \mathrm{LMP}^{-}$cells in the parental Awia-BL line gradually increased with continued passage, perhaps reflecting a selection process for cell survival that also may have been occurring in vivo. The isolation of rare EBVnegative Awia-BL clones from very early passage cultures of the Awia-BL line is particularly intriguing in that regard. Although these cells could have arisen through early loss of the virus genome in vitro, they also could be remnants of a c-myc translocationpositive tumor progenitor population that subsequently acquired EBV relatively late in tumor development.

As to how EBV contributes to BL pathogenesis, the present findings greatly strengthen the evidence that EBV's role is antiapoptotic rather than growth-transforming. Thus, in AwiaBL, Latency I infection confers a small but significant protective effect, whereas $\mathrm{Wp}$ restriction, where cells also express the EBNA3 proteins, confers much stronger protection. Interestingly, further broadening of EBNA expression to include EBNA2 did not give any additional survival advantage even though EBNA2, through binding the cellular protein Nur77, has been reported to protect cells from certain apoptotic triggers (25, 26). In fact, the EBNA2 ${ }^{+} / \mathrm{LMP}^{-}$- Awia-BL clones were slightly more susceptible to apoptosis than were $\mathrm{Wp}$-restricted clones, a modification that may reflect the competition seen in other contexts between the EBNA2 and EBNA3 proteins $(27,28)$.

Deregulated expression of the translocated c-myc allele is clearly the major determinant of BL cell proliferation (11). We suggest that tumor evolution selects for forms of EBV latency that can counteract the proapoptotic influence of the c-myc-driven growth program yet which, unlike full Latency III infection $(11,12)$, are compatible with maintenance of that program. The present data show that this condition can be achieved in at least three ways. Latency I infection, despite offering the smallest survival advantage, is seen most frequently in BL probably because Qp is the EBNA promoter used naturally in EBV-infected germinal centre cells (29), the population from which many cases of this tumor are thought to arise (30). Wp restriction offers a much more significant survival advantage but is seen only in a minority of tumors, presumably because it requires a rare event, infection of the tumor progenitor cell with an EBNA2 gene-deleted mutant virus, to be achieved. The novel EBNA2 ${ }^{+} / \mathrm{LMP}^{-}$form of infection also offers a strong survival advantage but appears to be very rare, presumably reflecting the very special conditions required to sustain expression of all six EBNAs without activating the full EBV transforming program and, thereby, compromising c-myc expression.

\section{Materials and Methods}

Cell Lines. The Awia-BL cell line, derived from an ovarian tumor in a 12-year-old Ugandan female, was established in vitro and single-cell cloned as described in ref. 14. The standard Latency I cell line Rael-BL carrying WT episomal genomes only, and the Wp-restricted Sal-BL, Oku-BL, and Ava-BL lines carrying WT and EBNA2-deleted episomes, are described in ref. 13. The standard LCLs X50-7 (type 1 EBV) and C2+BL16 (type 2 EBV) LCLs are described in ref. 5. The Awia tr-LCL was made by inducing virus replication in the Awia-BL line and rescuing transforming virus by infection of normal B cells (5). All BL lines and LCLs were maintained as described in ref. 13.

EBV Genome Analysis. DNA was extracted from $5 \times 10^{6}$ cells by using a DNEasy Tissue Kit (Qiagen, Valencia, CA) according to the manufacturer's instructions, and $5 \mathrm{ng}$ of DNA was assayed for EBV genome load by quantitative PCR amplification by using primer/probe combinations specific for the EBV DNA polymerase (pol) locus (31) and multiplexed by using primer/ probe combinations specific for the human $\beta 2$ microglobulin (B2m) gene $(32,33)$. Subsequently, $400 \mathrm{ng}$ of DNA was PCRamplified as described in ref. 34 by using the E2seq 1 and 2 primers within the EBNA2 gene to detect the WT genomes. After gel electrophoresis, the samples were transferred to a nitrocellulose membrane by Southern blotting and probed with a ${ }^{32} \mathrm{P}$ end-labeled radioactive EBNA2 probe 5'-AGGGATGCCTGGACACAAGA-3' (B95.8 coordinates 48810-48829). To detect the EBNA2 gene-deleted genome, $400 \mathrm{ng}$ of DNA was amplified by using primers 5'-GGGCCAGAGGTAAGTGGACTTT-3' (B95.8 coordinates 14611-14632) and 5'CCCACCTGGTGACACACCTTAA-3' (B95.8 coordinates 52938-52917), and the reaction cycled 35 times through $95^{\circ} \mathrm{C}$ for $60 \mathrm{sec}, 55^{\circ} \mathrm{C}$ for $60 \mathrm{sec}$, and $72^{\circ} \mathrm{C}$ for $120 \mathrm{sec}$. After Southern blotting, the membrane was hybridized with a radiolabeled probe derived from the BamHI H region 5'-GTGTCATTTTAGCCCGTTGG-3' (B95.8 coordinates 52891-52910). FISH analysis was carried out as described in ref. 35 by using an EBV 
genome-specific probe generated by nick translation of the EBV cosmid $\mathrm{cm} 302-21$ of $51 \mathrm{~kb}(36)$, alongside a spectrum-orangelabeled probe specific either for the centromeric region of chromosome 4 (CEP4) or for the telomeric region of chromosome 5 (TelVysion 5q) (Vysis. Des Plaines, IA).

EBV Gene Expression. Total RNA extraction and cDNA synthesis were carried out as described in ref. 14. Quantitative RT-PCR assays specific for Wp-initiated, Cp-initiated, EBNA2, LMP1 and LMP2 mRNAs, and Qp-initiated (BamHI Q-U-K spliced) EBNA1 latent transcripts are described in Supporting Text and Table 1, which are published as supporting information on the PNAS web site. Levels of transcription in test cells are expressed relative to that seen in an appropriate positive control cell line which was assigned an arbitrary value of one; these control lines are described in Supporting Text. Blotting was carried out by using mAbs to EBNA1 (1H4), EBNA2 (PE2), LMP1 (CS1-4) (14), c-Myc [(C-8): sc41; Santa Cruz Biotechnology, Santa Cruz, CA) and $\beta$-actin (AC-15, Sigma, St. Louis, MO) and a polyclonal antibody specific for EBNA3A (Exalpha Biologicals, Maynard, MA).

1. Kuppers R (2003) Nat Rev Immunol 3:801-812.

2. Rickinson AB, Kieff E (2001) in Fields Virology, eds Knipe DM, Howley PM (Lippincott, Philadelphia), Vol II, pp 2575-2627.

3. Kieff E, Rickinson A (2001) in Fields Virology, eds Knipe DM, Howley PM (Lippincott, Philadelphia), Vol II, pp 2511-2573.

4. Magrath I (1990) Adv Cancer Res 55:133-270.

5. Rowe M, Rowe DT, Gregory CD, Young LS, Farrell PJ, Rupani H, Rickinson AB (1987) EMBO J 6:2743-2751.

6. Kennedy G, Komano J, Sugden B (2003) Proc Natl Acad Sci USA 100:1426914274.

7. Komano J, Sugiura M, Takada K (1998) J Virol 72:9150-9156.

8. Komano J, Maruo S, Kurozumi K, Oda T, Takada K (1999) J Virol 73:98279831.

9. Ruf IK, Rhyne PW, Yang C, Cleveland JL, Sample JT (2000) J Virol 74:10223-10228.

10. Ruf IK, Rhyne PW, Yang H, Borza CM, Hutt-Fletcher LM, Cleveland JL, Sample JT (1999) Mol Cell Biol 19:1651-1660.

11. Polack A, Hortnagel K, Pajic A, Christoph B, Baier B, Falk M, Mautner J, Geltinger C, Bornkamm GW, Kempkes B (1996) Proc Natl Acad Sci USA 93:10411-10416.

12. Pajic A, Staege MS, Dudziak D, Schuhmacher M, Spitkovsky D, Eissner G, Brielmeier M, Polack A, Bornkamm GW (2001) Int J Cancer 93:810-816.

13. Kelly G, Bell A, Rickinson A (2002) Nat Med 8:1098-1104.

14. Kelly GL, Milner AE, Tierney RJ, Croom-Carter DS, Altmann M, Hammerschmidt W, Bell AI, Rickinson AB (2005) J Virol 79:10709-10717.

15. Shannon-Lowe C, Baldwin G, Feederle R, Bell A, Rickinson A, Delecluse HJ (2005) J Gen Virol 86:3009-3019.

16. Gregory CD, Dive C, Henderson S, Smith CA, Williams GT, Gordon J, Rickinson AB (1991) Nature 349:612-614.

17. Pelengaris S, Khan M, Evan G (2002) Nat Rev Cancer 2:764-776.

18. Shimizu N, Tanabe-Tochikura A, Kuroiwa Y, Takada K (1994) J Virol 68:6069-6073.

19. Niedobitek G, Agathanggelou A, Rowe M, Jones EL, Jones DB, Turyaguma P, Oryema J, Wright DH, Young LS (1995) Blood 86:659-665.
Apoptosis Assays. BL cells at $3 \times 10^{5}$ cells per $\mathrm{ml}$ were exposed at $37^{\circ} \mathrm{C}$ to known concentrations of ionomycin (Sigma) or anti-IgM antibody (ICN Flow) for 24, 48, 72, and $96 \mathrm{~h}$, then dually stained with $25 \mathrm{nM}$ Syto 16 (Molecular Probes Europe, Leiden, The Netherlands) and $2.5 \mu \mathrm{g} / \mathrm{ml}$ propidium iodide (Sigma) and analyzed immediately by flow cytometry as described in refs. 37 and 38. Data for 10,000 cells was collected for each cell line. Confirmatory apoptosis assays were carried out by using flow cytometry and fluorescence-based detection of activated caspases (CaspaLux; OncoImmunin, Gaithersburg, MD).

We thank Debbie Croom-Carter for excellent technical assistance, Paul Murray and Joanne Flavell for staining of tumor sections, Graham Fews and Mike Griffiths (Birmingham Women's Hospital, Birmingham, U.K.) for cytogenetic analyses, David Lloyd for assistance creating flow cytometer profiles, and Wolfgang Hammerschmidt and Marcus Altmann (GSF-National Research Center for Environment and Health, Munich, Germany) for the recombinant EBNA2-deletion mutant. This work was supported by Cancer Research UK.

20. Nanbo A, Inoue K, Adachi-Takasawa K, Takada K (2002) EMBO J 21:954-965. 21. Abbot SD, Rowe M, Cadwallader K, Ricksten A, Gordon J, Wang F, Rymo L, Rickinson AB (1990) J Virol 64:2126-2134.

22. Wang F, Tsang SF, Kurilla MG, Cohen JI, Kieff E (1990) J Virol 64:3407-3416.

23. Zimber-Strobl U, Suentzenich KO, Laux G, Eick D, Cordier M, Calender A Billaud M, Lenoir GM, Bornkamm GW (1991) J Virol 65:415-423.

24. Jochner N, Eick D, Zimber-Strobl U, Pawlita M, Bornkamm GW, Kempkes B (1996) EMBO J 15:375-382.

25. Lee JM, Lee KH, Weidner M, Osborne BA, Hayward SD (2002) Proc Natl Acad Sci USA 99:11878-11883.

26. Lee JM, Lee KH, Farrell CJ, Ling PD, Kempkes B, Park JH, Hayward SD (2004) J Virol 78:12694-12697.

27. Johannsen E, Miller CL, Grossman SR, Kieff E (1996) J Virol 70:4179-4183.

28. Waltzer L, Perricaudet M, Sergeant A, Manet E (1996) J Virol 70:5909-5915.

29. Babcock GJ, Hochberg D, Thorley-Lawson AD (2000) Immunity 13:497-506

30. Kurth J, Hansmann ML, Rajewsky K, Kuppers R (2003) Proc Natl Acad Sci USA 100:4730-4735.

31. Gallagher A, Armstrong AA, MacKenzie J, Shield L, Khan G, Lake A, Proctor S, Taylor P, Clements GB, Jarrett RF (1999) Int J Cancer 84:442-448.

32. Summers KE, Goff LK, Wilson AG, Gupta RK, Lister TA, Fitzgibbon J (2001) J Clin Oncol 19:420-424.

33. Junying J, Herrmann K, Davies G, Lissauer D, Bell A, Timms J, Reynolds GM, Hubscher SG, Young LS, Niedobitek G, Murray PG (2003) Virology 306:236243.

34. Yao QY, Tierney RJ, Croom-Carter D, Cooper GM, Ellis CJ, Rowe M, Rickinson AB (1996) J Virol 70:4895-4903.

35. Delecluse HJ, Schuller S, Hammerschmidt W (1993) EMBO J 12:3277-3286.

36. Polack A, Hartl G, Zimber U, Freese UK, Laux G, Takaki K, Hohn B, Gissmann L, Bornkamm GW (1984) Gene 27:279-288.

37. Milner AE, Palmer DH, Hodgkin EA, Eliopoulos AG, Knox PG, Poole CJ, Kerr DJ, Young LS (2002) Cell Death Differ 9:287-300.

38. Schuurhuis GJ, Oberink JW, Brontje PM, Broxterman HJ (1997) Exp Hematol 25:754. 